\title{
Optimal delivery of follow-up care after surgery for Crohn's disease: current perspectives
}

\section{James P Campbell \\ Byron PVaughn}

Department of Gastroenterology, Hepatology and Nutrition, University of Minnesota, Minneapolis, MN, USA

This article was published in the following Dove Press journal:

Clinical and Experimental Gastroenterology

8 August 2016

Number of times this article has been viewed

\begin{abstract}
Despite improvements in medical therapies for Crohn's disease (CD), up to $70 \%$ of patients require surgery within 10 years of diagnosis. Surgery is not curative, and almost all patients will experience endoscopic recurrence, and many will go on to clinical recurrence. Identifying patients at high-risk of endoscopic recurrence and standardizing postoperative assessments are essential in preventing clinical recurrence of $C D$. In this review, we discuss the assessment, monitoring, and treatment of postoperative CD patients. We address the various individual risk factors as well as composite risk factors. Medications used for primary CD treatment can be used in the postoperative setting to prevent endoscopic or clinical recurrence with varying efficacy, although the cost-effectiveness of these approaches are not fully understood. Future directions for postoperative CD management include evaluation of newer biologic agents such as anti-integrin therapy and fecal microbiota transplant for prevention of recurrence. Development of a standard preoperative risk assessment tool to clearly stratify those at high-risk of recurrence is necessary to guide empiric therapy. Lastly, the incorporation of noninvasive testing into disease monitoring will likely lead to early detection of endoscopic recurrence that will allow for tailored treatment to prevent clinical recurrence.
\end{abstract}

Keywords: Crohn's disease, postoperative care, postoperative recurrence

\section{Introduction}

Crohn's disease (CD) is a chronic inflammatory disease of the small and large intestine that results in significant morbidity, specifically the need for surgery. Despite advances in medical therapy, up to $70 \%$ of patients with $\mathrm{CD}$ will require surgery within 10 years of diagnosis. ${ }^{1,2}$ Surgical resection is not curative, and approximately $70 \%$ of patients will have endoscopic recurrence at 1 year. The overall clinical recurrence rates following surgery are $20 \%$ and $30 \%$ at 1 and 3 years, respectively, although they clearly differ based on the presence of endoscopic recurrence. ${ }^{3-5}$ The most common surgical procedure for $\mathrm{CD}$ is bowel resection, specifically an ileocecectomy, occurring in $60 \%-70 \%$ of patients who require surgery. ${ }^{6-8}$ Historically, patients were only treated for clinical recurrence following surgery. However, with novel CD therapies and a paradigm change to utilize treatment to alter the course of the disease, there is a clear role for treatment before clinical recurrence. This can occur as empiric treatment for high-risk patients or tailored treatment based on endoscopic recurrence. The intent of these strategies is to prevent clinical recurrence and subsequent need for further surgical intervention.

Correspondence: James P Campbell 2320 Marshall Avenue, Apartment 410, Saint Paul, MN 55104, USA

Tel +l 6086985554

Fax + 6123013021

Email camp0795@umn.edu 
In this review, we outline the steps associated with the optimal care for postsurgical CD patients and review the evidence surrounding the available options in each step: risk stratification for postoperative recurrence, treating endoscopic recurrence to prevent clinical recurrence, monitoring for progression of disease, and achieving target-directed treatment goals. Although surgery for CD is a broad term, we will focus on ileocecal resections with an ileocolonic anastomosis, as the majority of literature is in this area. It is likely that these principles can be applied to other postoperative CD settings. There are three general strategies in the management of postoperative $\mathrm{CD}: 1)$ empiric treatment following surgery to prevent recurrence; 2) assessment and treatment of endoscopic recurrence to prevent clinical recurrence; and 3) treatment of clinical recurrence. Optimal treatment at this time should focus on preventing clinical recurrence and utilizing a combination of empiric treatment for high-risk individuals and routine assessment and treatment of endoscopic recurrence to prevent a clinical recurrence after surgery.

\section{Risk stratification}

Following surgery for CD, the initial question is: Does the patient require empiric therapy to prevent endoscopic and clinical recurrence? This decision is ideally made prior to surgery and should be done in conjunction with the surgical team. There is currently no validated risk model or score to stratify patients postoperatively; thus, the decision to label a patient "high-risk" and start empiric therapy needs to be individualized, taking into consideration the prior CD history and patients' preferences. Consistently identified risk factors for both endoscopic and clinical recurrence include smoking, ${ }^{9,10}$ history of prior surgery or resection, ${ }^{11,12}$ perforating disease (defined as occurrence of bowel perforation, intra-abdominal fistulas, or inflammatory masses and/or abscesses, in accordance with the Montreal B3 classification for penetrating disease behavior, but does not include isolated perianal fistula), ${ }^{13-15}$ and myenteric plexitis. ${ }^{16-18}$ Other factors with supportive data and variable use in risk assessment models proposed thus far include length of resection, ${ }^{19,20}$ disease location (such as perianal disease), ${ }^{19}$ and shorter disease duration. ${ }^{21}$ Newly investigated risk factors that require further validation include microbiota diversity, ${ }^{22,23}$ type of anastomosis, ${ }^{11,24}$ histological disease ${ }^{25}$ (including decreased lymphatic vessel density, ${ }^{26}$ granulomas in mesenteric lymph nodes, ${ }^{27}$ and $\mathrm{CD} 8{ }^{+} \mathrm{T}$-cells in blood and ileal mucosa $\left.{ }^{28}\right)$, and visceral fat area on computed tomography $(\mathrm{CT})^{29}$ (Table 1$)$.
Table I Risk factors for endoscopic and clinical recurrence after surgery for Crohn's disease

\begin{tabular}{lll}
\hline $\begin{array}{l}\text { Confirmed risk } \\
\text { factors }\end{array}$ & $\begin{array}{l}\text { Probable risk } \\
\text { factors }\end{array}$ & $\begin{array}{l}\text { Investigational risk } \\
\text { factors }\end{array}$ \\
\hline Smoking & Shorter disease & Genetic mutations \\
Perforating disease & duration & Histological disease factors \\
Previous surgery & Disease location & Imaging findings \\
Myenteric plexitis & Extent of disease & Microbial diversity \\
& Granulomas & Type of anastamosis \\
\hline
\end{tabular}

Table 2 Models for defining patients at high-risk for postoperative Crohn's disease recurrence proposed by various authors

\begin{tabular}{ll}
\hline Model & Characteristics of high-risk patients \\
\hline De Cruz et al (2015) & $\begin{array}{l}\text { One or more of: smoking, perforating } \\
\text { disease, or previous resection }\end{array}$ \\
Vaughn et al (2014) & $\begin{array}{l}\text { Two or more of: smoking, perforating } \\
\text { disease, previous resection, or myenteric } \\
\text { plexitis }\end{array}$ \\
Regueiro et al (2009) & $\begin{array}{l}\text { Perforating disease or history of multiple } \\
\text { surgeries } \\
\text { Two or more of: smoking, previous } \\
\text { Buisson et al }(2012)^{33}\end{array}$ \\
& $\begin{array}{l}\text { resection, perforating disease, perianal } \\
\text { location, or extensive bowel resection }\end{array}$ \\
\hline
\end{tabular}

Various definitions of "high"-risk exist based on the number of risk factors present (Table 2). In the postoperative Crohn's endoscopic recurrence (POCER) randomized trial, which compared patients who received systematic endoscopy after surgery (active care) to no endoscopy (standard care), high-risk patients were defined as having one or more of the following risk factors: smoking, perforating disease, or previous resection. ${ }^{30}$ Using these criteria, $83 \%$ of patients enrolled were high-risk. Unfortunately, the definition for "high-risk" is arbitrary and will remain so until a prospective validated prediction tool is developed. Regueiro ${ }^{31}$ divides risk into three categories: low-risk includes those with $\mathrm{CD}$ duration $>10$ years, a short stricture $(<10 \mathrm{~cm})$, and first surgery; moderate risk includes patients with disease $<10$ years duration, active inflammation, and intestinal narrowing, but without perforating or fistulizing disease or a history of multiple surgeries; and high risk as having perforating disease and history of previous surgery. ${ }^{31,32}$ Buisson et $\mathrm{al}^{33}$ classify risk based on the European Crohn's and Colitis Organization definition of established risk factors for smoking, prior intestinal surgery, perforating disease behavior, perianal location of disease, and extensive small bowel resection. ${ }^{34}$ In their model, patients are stratified by number of risk factors for initial medical therapy after surgery, resulting in a three-tiered risk model where no risk factors was considered low-risk, one risk factor was moderate risk, and greater than or equal to two risk factors was high risk. In our practice, we typically define high-risk 
as those with two or more of the confirmed risk factors listed in Table 1. However, this is not based on strong evidence, but rather our own experience and interpretation of the evidence. In many situations, we will discuss empiric therapy for patients with only one risk factor and choose to monitor patients with two or more risk factors closely. In general, as our practice evolves, we rely more on an early (within 6 months postoperatively) colonoscopy to identify endoscopic recurrence to tailor therapy, rather than just preoperative or operative risk factors. Cleary, this is an area with much variability that will require more research to guide our clinical practice.

Initial risk stratification is important, as empiric treatment should be tailored to the risk assessment. Models based on established risk factors can be used for guiding initial therapy; however, ultimately, therapy should be individualized based on specific patient characteristics and prior treatment history.

\section{Empiric treatment for high-risk individuals}

Generally, patients who are high-risk (defined by the treating physician) are treated empirically to prevent recurrence of CD. Deciding what medication to start requires knowledge of the efficacy of each agent as well as the patient's personal history of CD therapy. Failure of a class of medications preoperatively does not necessarily mean that medication class cannot be used in the postoperative setting, although there should be a rationale for a retrial and close assessment to determine efficacy. Almost all classes of therapy for CD treatment have been studied for the prevention of postoperative recurrence. Newer medications such as vedolizumab and novel therapies such as fecal microbiota transplant (FMT) may provide more targeted and potentially safer approaches, although clinical data are not yet available.

\section{Mesalamine}

Mesalamine agents are appealing, as they are low cost and have a favorable safety profile. In a meta-analysis, mesalamine was associated with a reduction in clinical recurrence in comparison to placebo (relative risk $[\mathrm{RR}]=0.60 ; 95 \%$ confidence interval $[\mathrm{CI}]=0.37-0.88$ ) and a trend toward, although not statistically significant, reduction of endoscopic recurrence $(\mathrm{RR}=0.67 ; 95 \% \mathrm{CI}=0.39-1.08) .{ }^{35}$ This was similar to a prior meta-analysis that found decreased clinical as well as severe (Rutgeerts score $\geq i 3$ ) endoscopic recurrence in patients on mesalamine in comparison to placebo, however, not a reduction in all endoscopic recurrence. ${ }^{36}$ Given the lack of efficacy for preventing endoscopic recurrence, mesalamines should likely not be routinely used as empiric treatment.

\section{Thiopurines}

Azathioprine and 6-mercaptopurine are efficacious in reducing clinical and endoscopic recurrence of postoperative CD. In two trials comparing thiopurines to placebo, they reduced endoscopic recurrence, and pooled data revealed reductions in clinical and endoscopic recurrence. ${ }^{36-38}$ In a meta-analysis of studies comparing thiopurines to mesalamine, both were equally effective at maintaining clinical remission; however, thiopurines were more effective at reducing endoscopic recurrence for the first year $(\mathrm{RR}=0.71) .{ }^{39}$ However, in a randomized trial, $22 \%$ of participants had to stop thiopurines due to side effects or medication intolerance. ${ }^{40}$ In a network meta-analysis, thiopurines were associated with reduced clinical $(\mathrm{RR}=0.36 ; 95 \% \mathrm{CI}=0.17-0.63)$ and endoscopic $(\mathrm{RR}$ $=0.33 ; 95 \% \mathrm{CI}=0.13-0.68)$ recurrence. ${ }^{35}$ Given this, thiopurines are likely beneficial in moderate- to high-risk patients who are able to tolerate them without significant side effects.

\section{Probiotics}

Probiotics are an attractive option given the data that suggest that the microbiome is altered in disease recurrence, and they also have minimal side effects. However, there are limited data on their efficacy. One randomized trial of VSL\#3 in postoperative patients found a trend toward, but not statistically significant, reduction in endoscopic recurrence compared to placebo. ${ }^{41}$ However, pooled data from randomized trials investigating various probiotic formulations failed to show any difference with regard to clinical or endoscopic recurrence. ${ }^{36}$ At this time, there does not appear to be sufficient data to support the routine use of probiotics as empiric treatment for reducing the risk of recurrence.

\section{Antibiotics}

Nitroimidazole antibiotics can reduce the rate of clinical and endoscopic recurrence. Metronidazole was associated with a reduction in risk of clinical and endoscopic recurrence in a randomized clinical trial and systematic review, ${ }^{36,42}$ with a greater effect when used in combination with azathioprine in a randomized trial. ${ }^{37}$ Ornidazole was also associated with reduced clinical and endoscopic recurrence risk. ${ }^{43}$ However, the effects of antibiotics may be limited if therapy with another agent is planned, as the addition of metronidazole to azathioprine did not reduce the risk of endoscopic recurrence in a randomized pilot study. ${ }^{44}$ In addition, there are also concerns about the tolerability and safety when used 
as long-term therapy. In the POCER trial, all patients were prescribed a 3-month course of metronidazole regardless of risk. Seventy percent of patients tolerated full-dose metronidazole, $8 \%$ tolerated a reduced dose, and $20 \%$ had to stop metronidazole due to intolerance. ${ }^{30}$ However, a decision analysis model identified antibiotics as the most cost-effective prophylactic treatment for reducing clinical postoperative recurrence. ${ }^{45}$ Therefore, if tolerated, routine treatment with a nitroimidazole antibiotic may be a reasonable approach for patients in the postoperative setting.

\section{Anti-tumor necrosis factor}

Anti-tumor necrosis factor (anti-TNF) therapy, specifically infliximab and adalimumab, is more effective than other therapies at reducing clinical and endoscopic recurrence. In meta-analyses of randomized controlled trials evaluating empiric drug therapy in postoperative patients, anti-TNF therapy was the most effective therapy in reducing clinical (RR or odds ratio [OR] of 0.04-0.36) and endoscopic (RR or OR of $0.01-0.16$ ) recurrence..$^{35,46,47}$ In a network metaanalysis of 15 trials, anti-TNFs were associated with the largest reduction in endoscopic and clinical recurrence in comparison to thiopurines, mesalamine, and placebo. ${ }^{48}$

In addition to improved short-term outcomes compared to other therapies, anti-TNFs, in particular infliximab, have demonstrated long-term efficacy. In the PREVENT trial, a randomized trial of 297 patients comparing infliximab $5 \mathrm{mg} / \mathrm{kg}$ to placebo in the postoperative setting, there was a trend toward the primary end point of reduced clinical recurrence in infliximab-treated patients at weeks 76 and 104 , although this was not statistically significant $(P=0.097$ and 0.098 , respectively). ${ }^{49}$ However, there was significantly less endoscopic recurrence in infliximab-treated patients in comparison to placebo $(P<0.001)$. Multiple other trials have demonstrated infliximab to be efficacious at 1 year, ${ }^{50,51}$ and long-term data at 2 and even 5 years demonstrated reduced endoscopic recurrence, longer time to recurrence, and less need for additional surgery. ${ }^{52}$ In addition to endoscopic remission, one pilot study found that infliximab prevented histologic recurrence of $\mathrm{CD}$ compared to azathioprine at 1 year. ${ }^{53}$

Adalimumab appears to be effective to a similar degree as infliximab. In pilot and retrospective analyses, adalimumab was effective at reducing clinical and endoscopic recurrence at rates comparable to infliximab. ${ }^{54-56}$ An analysis of POCER data found that adalimumab was more effective at reducing endoscopic recurrence than thiopurines. ${ }^{57}$ In a randomized trial of 51 patients comparing adalimumab, azathioprine, and mesalamine, adalimumab was associated with reduced clinical and endoscopic recurrence. ${ }^{58}$

On the basis of the available data, anti-TNFs appear to be the most efficacious for reducing both clinical and endoscopic recurrence, and thus are likely indicated in highrisk patients, especially those who are unable to tolerate thiopurines. However, there are concerns about the cost of anti-TNFs. Cost-effectiveness models did not demonstrate intravenous anti-TNFs to be cost-effective at 1 year postoperatively. ${ }^{45,59}$ Notably, injectable anti-TNFs were not included in this analysis because there was insufficient data at that time. Finally, an anti-TNF may not be the ideal therapy if the patient was on an anti-TNF preoperatively. A retrospective analysis of 57 patients revealed that exposure to two or more anti-TNFs prior to surgery was associated with higher rates of endoscopic and clinical recurrence. ${ }^{60}$ While not an absolute contraindication for anti-TNF therapy, the decision to use an anti-TNF in the postoperative setting must take into consideration prior anti-TNF use and the reason for cessation. Likely, the reason for prior anti-TNF failure will predict the benefit of retreatment in the postoperative setting. ${ }^{61}$

\section{Combination therapy}

Combination therapy in $\mathrm{CD}$ typically refers to the concomitant use of a thiopurine and an anti-TNF. In treatment naïve $\mathrm{CD}$, this combination of medications is clearly superior to monotherapy at inducing and maintaining remission. ${ }^{62} \mathrm{How}-$ ever, following surgery, it is not clear why two immunosuppressive medications are needed. In a retrospective analysis of 41 patients, the addition of azathioprine to infliximab was correlated with the continuation of infliximab treatment, suggesting there may be benefit in reducing immunogenicity to infliximab. ${ }^{63}$ In a separate retrospective analysis of 168 patients, there was no difference in endoscopic recurrence within 12 months after surgery in patients treated with antiTNF monotherapy or anti-TNF plus thiopurine combination therapy. ${ }^{64}$ This limited data suggest monotherapy with an anti-TNF is likely sufficient and an immunomodulator may be used to decrease the immunogenicity in certain patients.

Other drug combinations have also been studied in the postoperative setting. Two studies have evaluated the combination of azathioprine for 1 year and metronidazole for 3 months in comparison to metronidazole monotherapy or azathioprine monotherapy. D'Haens et $\mathrm{al}^{37}$ found a reduction in endoscopic recurrence with the addition of azathioprine for 1 year to metronidazole for 3 months in comparison to metronidazole for 3 months alone. Manosa et $\mathrm{al}^{44}$ did not find a difference in endoscopic recurrence in patients on 
azathioprine for 1 year, regardless of whether metronidazole for 3 months was added. Thus, the addition of metronidazole appears unlikely to have a benefit when the patient is already on an immunomodulator. The Adalimumab on Preventing Postsurgical Recurrence on Crohn's Disease (APPRECIA) study is a randomized trial comparing metronidazole plus azathioprine to metronidazole plus adalimumab, and it has not been published yet (NCT01564823).

\section{Timing of empiric therapy}

The optimal timing of initiating therapy is not entirely clear as trials have shown mixed results when comparing systematic empiric therapy versus endoscopy-driven treatment. However, early initiation after surgery (within 4 weeks) has been advocated for high-risk patients given the high rate of endoscopic recurrence and the association with future clinical recurrence, while low-risk patients may benefit from close endoscopic monitoring at 6 months and treatment based on endoscopic findings. Two studies suggest initiating therapy later may not have an adverse effect on outcomes. A retrospective analysis of postoperative CD patients did not identify a difference in endoscopic, clinical, or surgical recurrence in patients started on therapy early ( $<4$ weeks) or late ( $>4$ weeks) postoperatively. ${ }^{65}$ However, the late group was treated more commonly with anti-TNFs than the early group. In another retrospective analysis of 199 patients, clinical recurrence rates were lower in patients treated with immediate postoperative therapy versus therapy driven by endoscopy within 6 months of surgery on univariate analysis, however, not on multivariate analysis when controlled for length of follow-up. ${ }^{66}$ In the authors' opinion, when the decision to treat empirically is made, therapy should be initiated within 2-4 weeks after an uncomplicated surgery so as to prevent early endoscopic inflammatory changes. It is essential that the decision be made in conjunction with the surgical team to ensure the safety of this approach.

\section{Postoperative therapeutic drug monitoring}

Similar to the preoperative setting, in the postoperative setting serum drug concentrations are associated with endoscopic and clinical response. For treatment of endoscopic recurrence, a post hoc analysis of a randomized trial found that higher 6-thioguanine levels were associated with endoscopic improvement in patients with severe postoperative endoscopic recurrence. ${ }^{67}$ Similarly, median adalimumab trough levels were lower in patients with clinical or endoscopic recurrence in another post hoc analysis. ${ }^{68}$ Two trials of proactive infliximab concentration monitoring have demonstrated improved remission rates and a longer duration of infliximab use with monitoring. ${ }^{69,70}$ In both instances, approximately $40 \%$ of the study population consisted of postoperative $\mathrm{CD}$ patients, and there was a clinical benefit in monitoring infliximab concentrations. Although prospective studies specific to postoperative CD need to be performed, these data suggest that optimizing an anti-TNF to a therapeutic concentration will improve the outcomes for postoperative CD patients similarly to preoperative ones.

Dosing anti-TNFs based on drug concentration may also allow for lower doses in the postoperative setting. In this case, the bulk of inflammation is surgically removed, which likely decreases the metabolism and/or stool loss of the anti-TNF. ${ }^{71}$ In a prospective cohort study of twelve patients, low-dose infliximab $(3 \mathrm{mg} / \mathrm{kg})$ was effective at maintaining endoscopic remission at 1 year. ${ }^{72}$ In a small comparison study of a subset of five of those patients on low-dose infliximab (3 $\mathrm{mg} / \mathrm{kg}$ ) compared to patients on standard dosing $(5 \mathrm{mg} / \mathrm{kg})$, all remained in endoscopic remission at 30 months after surgery. ${ }^{73}$ Thus, therapeutic concentrations of an anti-TNF may be able to be achieved at a lower dose, while on the other hand, low concentrations could be a surrogate marker for disease recurrence and increased drug turnover. Given that the inflammatory burden is lower in the postoperative setting, and fecal losses of anti-TNFs are presumably minimized, deescalation of anti-TNF dose based on trough concentration is an appealing strategy that has not been directly studied.

\section{Tailored treatment for endoscopic recurrence Detecting recurrent disease Endoscopy}

Identifying endoscopic recurrence and tailoring therapy to prevent clinical recurrence is becoming the standard of postoperative care for lower risk patients. Detecting endoscopic recurrence is important because it can predict clinical recurrence as well as separate out recurrent $C D$ from noninflammatory causes of diarrhea, such as infections, bile salt diarrhea, and bacterial overgrowth, as symptoms may correlate poorly to inflammation. ${ }^{74}$ Endoscopy is currently the gold standard for diagnosis of recurrent disease, and ileal recurrence can be defined based on the Rutgeerts score: ${ }^{5}$ i0 - no lesions, i1 - less than or equal to five aphthous lesions, i2 - greater than five aphthous lesions with normal intervening mucosa, i3 - diffuse aphthous ileitis with diffusely inflamed mucosa, i4 - diffuse inflammation with large ulcers, nodules, and/or narrowing. A score of $\mathrm{i} 2$ or greater is considered endoscopic 
recurrence. Recurrent mucosal disease after surgery precedes clinical symptoms, and its severity predicts subsequent clinical disease..$^{5,75}$

Capsule endoscopy may be useful as an adjunctive imaging study that was relatively equal to endoscopy findings in a recent review and may detect lesions missed by or unreachable with endoscopy. ${ }^{76}$ However, capsule endoscopy has only been studied in small patient numbers and likely should only be used to assess for recurrence when endoscopy is contraindicated or unable to reach the surgical anastomosis. Given the poor predictability of clinical symptoms for endoscopic recurrence, and the invasiveness and cost of endoscopy, there has been interest in using surrogate testing, such as imaging and biomarkers, to monitor for disease recurrence.

\section{Imaging}

Various imaging modalities hold promise as noninvasive methods for detecting postoperative recurrence, including CT, magnetic resonance imaging (MRI), and ultrasound. Enterography, either by CT or MRI, is both sensitive and specific for recurrent CD. ${ }^{77-79}$ Deciding between either test is based on concern for repeated ionizing radiation and the local radiologic expertise. Enteroclysis (standard, CT, or MRI) is also sensitive, specific, and accurate compared to endoscopy, although it is time and labor intensive and likely has little role if CT or MRI enterography is available. ${ }^{80-82}$ Other imaging techniques, such as ultrasonography, have demonstrated promise in research settings, but lack widespread clinical expertise at this time. ${ }^{83}$ Imaging can be quite helpful as a noninvasive marker of disease and provides additional information of other areas of the bowel. However, given the well-validated use of the Rutgeerts score and the ability to take biopsies, we prefer endoscopy when able to assess initial postoperative recurrence.

\section{Biomarkers}

Serum and fecal biomarkers have been studied as surrogate markers for inflammation in $\mathrm{CD}$, and in the postoperative setting, may play an important role in detecting recurrence and selecting patients for endoscopy. Fecal calprotectin (FC) levels correlate with endoscopic findings in CD before surgery, and their utility in monitoring for endoscopic recurrence has recently become of interest. In multiple small studies utilizing different FC cutoffs postoperatively, levels were associated with endoscopic activity. ${ }^{84-86}$ In a meta-analysis of ten studies with 613 patients with FC measured postoperatively, the pooled sensitivity and specificity for detecting endoscopic recurrence were $0.82(95 \% \mathrm{CI}=0.73-0.89)$ and $0.61(95 \%$
$\mathrm{CI}=0.51-0.71)$, respectively, and positive and negative likelihood ratios were $2.11(95 \% \mathrm{CI}=1.68-2.66)$ and $0.29(95 \%$ $\mathrm{CI}=0.197-0.44)$, respectively. ${ }^{87}$ In a prospective observational study, FC levels were significantly higher in patients with endoscopic recurrence, with a negative predictive value of $93 \%$ at a cutoff of $<100 \mu \mathrm{g} / \mathrm{g}$, which per the authors would have resulted in $30 \%$ fewer endoscopies in their cohort. ${ }^{88}$ An analysis of data from the POCER trial found that FC levels correlated with endoscopic recurrence, whereas serum C-reactive protein (CRP) and Crohn's disease activity index did not. ${ }^{89}$ In this study, a cutoff of FC $>100 \mu \mathrm{g} / \mathrm{g}$ identified patients with endoscopic recurrence with $89 \%$ sensitivity and $58 \%$ specificity, and the negative predictive value of a FC of $<100 \mu \mathrm{g} / \mathrm{g}$ was $91 \%$. Based on this cutoff, colonoscopy would not have been needed in $47 \%$ of patients without endoscopic recurrence, but with the tradeoff of missing $11 \%$ of patients with endoscopic recurrence. ${ }^{90}$ Based on these studies, our practice is to correlate $\mathrm{FC}$ with endoscopy. If they correlate well, then FC can be used to monitor for future recurrence or response to therapy after an initial endoscopy.

Serum inflammatory markers, such as CRP, have also been studied as a biomarker for recurrence, however, without strong data in support of their efficacy in detecting recurrence. Small studies have not demonstrated a consistent correlation between CRP and endoscopic recurrence, potentially owing to the inflammatory disease burden in postoperative patients not being large enough to cause a significant elevation. ${ }^{73,74,88}$ Based on the inconsistent data, CRP should not be used solely as a marker for monitoring recurrence.

\section{Treating endoscopic recurrent disease: treating-to-target}

Treatment of endoscopic recurrence is important given the high rate of progression to clinical recurrence. Two studies have specifically addressed treatment of endoscopic recurrence. Sorrentino et $\mathrm{a}^{11}$ investigated 24 patients with endoscopic recurrence treated with infliximab or mesalamine and showed that those treated with infliximab were more likely to be in endoscopic remission at 54 weeks ( $54 \%$ versus $0 \%$ ). In a pilot study by Yamamoto et al, ${ }^{92}$ patients treated with infliximab for endoscopic recurrence at 6 months postoperatively were more likely to have improved endoscopic inflammation and be in clinical remission as compared to azathioprine- or mesalamine-treated patients.

The concept of "treat-to-target" has gained importance in treating $\mathrm{CD}$, specifically targeting to endoscopic mucosal healing. ${ }^{93}$ The main therapeutic target identified by the Selecting Therapeutic Targets in Inflammatory Bowel Disease 
program for $\mathrm{CD}$ was mucosal healing on ileocolonoscopy, with use of cross-sectional imaging only in patients who cannot be adequately assessed with ileocolonoscopy. ${ }^{94}$ Serum and fecal markers of inflammation were not recommended targets, but instead adjunctive measures of inflammation to be used for monitoring.

Recent studies in postoperative patients have focused on adopting a treat-to-target strategy, specifically targeting endoscopic mucosal healing on systematic surveillance endoscopy postoperatively, and proactively stepping up therapy when endoscopic healing is not achieved. One retrospective study of patients who underwent systematic endoscopy and stepup therapy based on endoscopic findings revealed reduced clinical recurrence rates in those who underwent systematic endoscopy in comparison to those who did not. ${ }^{95}$ Another retrospective study found patients with endoscopic recurrence who were treated with step-up in therapy were less likely to have subsequent clinical recurrence. ${ }^{96}$ In a small prospective trial, no difference was seen in patients who received systematic azathioprine therapy versus endoscopydriven therapy. ${ }^{97}$ However, this trial was ended early due to low recruitment. In the POCER study, patients were treated initially based on the risk of recurrence and randomized to receive colonoscopy at 6 months postoperatively or no colonoscopy. Patients who received systematic colonoscopy and were treated with step-up therapy based on endoscopic findings had reductions in further clinical and endoscopic recurrence at 18 months after surgery. ${ }^{30}$ Based on these trials, a treat-to-target strategy should be the standard of care in treating postoperative $\mathrm{CD}$ patients. Currently, the optimal target is endoscopic healing, requiring surveillance endoscopy at regular intervals after surgery.

\section{Future therapies}

Potential upcoming areas of investigation for prevention and treatment of postoperative recurrence include newer pharmacologic agents, such as vedolizumab and ustekinumab, as well as treatments aimed at altering the microbiota, such as FMT. Vedolizumab is a gut-specific anti-integrin monoclonal antibody approved for the treatment of $\mathrm{CD}$ and ulcerative colitis and may be beneficial in the postoperative setting given its ability to reduce leukocyte trafficking in the gut and theoretically reduce early inflammatory changes. ${ }^{98-100}$ However, as of this writing, there are no studies assessing the use of vedolizumab to prevent endoscopic recurrence. FMT is currently being investigated in a clinical trial as an option for prevention of endoscopic recurrence (NCT02417974). The rationale behind FMT as a therapeutic modality stems from the benefit of antibiotics in postoperative $C D$, the fact that bacterial exposure via fecal stream is needed for recurrence, and divergent microbial diversity in patients with and without recurrence..$^{22,23,101}$

\section{Putting it into practice and future directions}

When a patient with CD undergoes surgery, the treating physician must first determine if they plan to empirically treat postoperatively to prevent recurrence or wait and assess for endoscopic recurrence and then treat. Empiric treatment should be individualized and incorporate prior failed therapy, the efficacy of the drug in the postoperative setting, and the cost of therapy. There is no one correct answer for prevention of recurrent disease, and this decision must be individualized. We recommend treatment based on initial risk assessment (Figure 1). We define high-risk patients based on having two or more of what we feel have been identified as the most consistent risk factors in the literature: smoking $(\mathrm{OR}=2.0)$, perforating disease $(\mathrm{OR}=1.5)$, prior resection $(\mathrm{OR}=1.8)$, and mesenteric plexitis $(\mathrm{OR}=1.9) .{ }^{102}$ However, we acknowledge that our risk stratification scheme is arbitrarily defined based on our opinion and not prospectively validated, underscoring the need for individualized assessment, as well as a standardized risk stratification tool. In all circumstances, smoking cessation must be strongly encouraged in all patients given its significant association with increased risk of clinical and endoscopic recurrence. If the decision is made not to treat to prevent postoperative recurrence, then we strongly advise routine postoperative assessment for endoscopic recurrence and treatment of endoscopic recurrence to prevent clinical recurrence. If noninvasive markers correlate with endoscopy, they can be used for monitoring; however, at this time, endoscopy remains the gold standard for assessing for endoscopic recurrence. Similar to prevention, the exact choice of treatment for endoscopic recurrence is unknown, but likely, the efficacy of treatment options mirrors that for clinical recurrence in $\mathrm{CD}$ patients who have not previously undergone surgery. We do not advise waiting for clinical recurrence in the postoperative setting. Regardless of the initial choice, any intervention (prevention or treatment) should be routinely assessed for efficacy at 6-12 months with ileocolonoscopy. Current monitoring is suboptimal, as $\mathrm{O}^{\prime}$ Connor et al ${ }^{103}$ reported in a retrospective analysis that $22.4 \%$ of patients had no endoscopic or radiologic evaluation of their disease over the course of a 32-month follow-up period, and only $42.8 \%$ of patients had postoperative colonoscopies. Among those who did, the average time to follow-up was 58 weeks. 


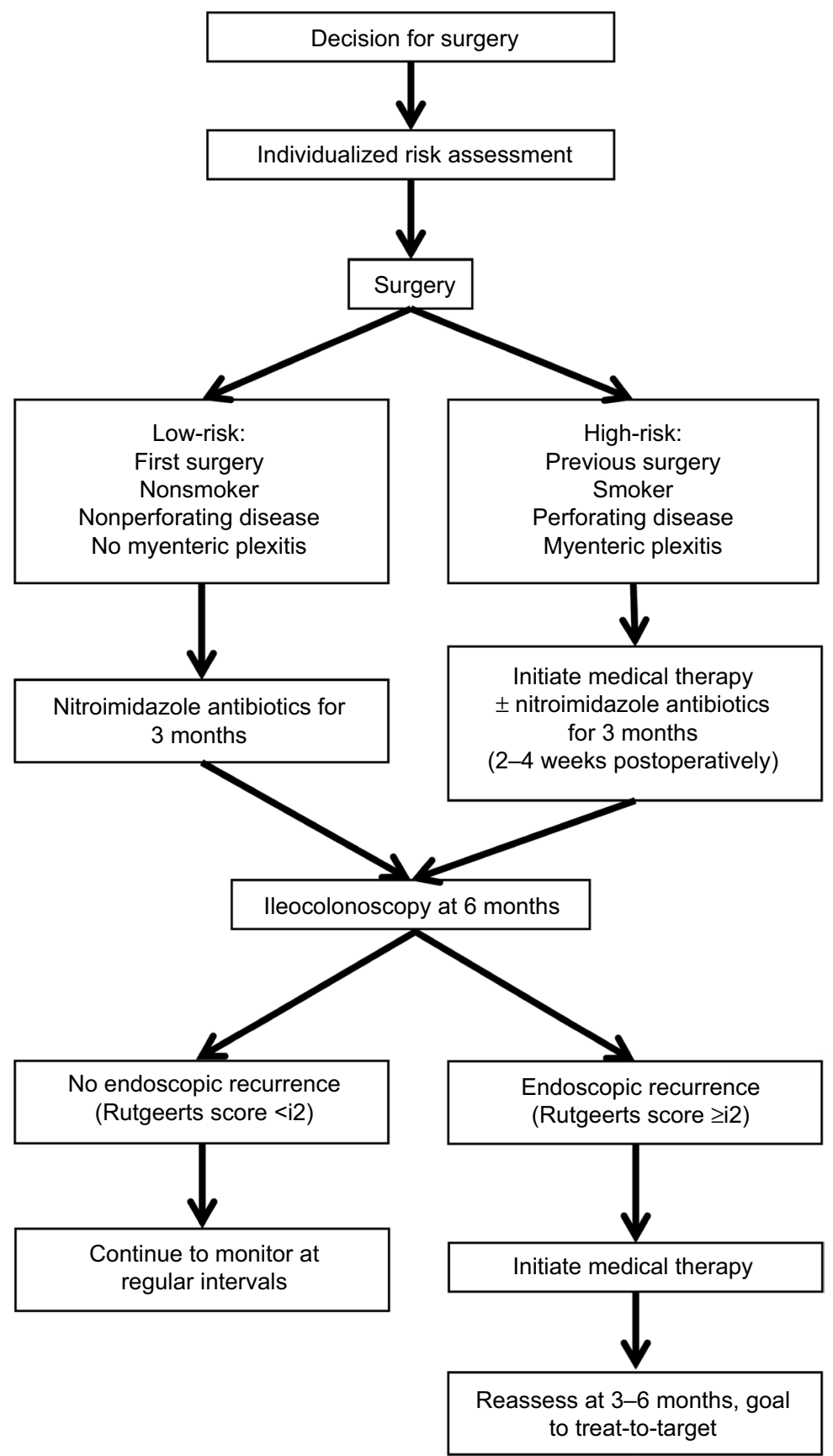

Figure I Early postoperative management following surgery for Crohn's disease.

Notes: The exact definition of high-risk is not standardized; however, we typically define high-risk as having two or more risk factors included in the "high-risk" box shown in the figure. Initiation of medical therapy is largely determined on the patient's prior history. Anti-TNFs are the most efficacious therapy for preventing endoscopic and clinical recurrence.

Abbreviation: TNFs, tumor necrosis factors.

Although we have progressed substantially in our understanding and management of postoperative $\mathrm{CD}$, many challenges still remain. At this time, there is no validated clinical tool to risk-stratify an individual's postoperative risk of recurrence. Such a tool would be very helpful in determining who to empirically treat following surgery versus who to survey for endoscopic recurrence and subsequently treat if present. Additionally, our understanding of biomarkers for disease recurrence is incomplete. Although FC is promising, determining exact cutoffs and prospective validation is needed before widespread use. Similarly, the exact role of various imaging modalities will need to be determined as well as assessed for cost-effectiveness compared to endoscopy. Therapeutic drug monitoring may allow for lower doses 
of anti-TNFs to be effectively utilized in the postoperative setting. Finally, novel therapies such as lymphocyte trafficking inhibition or FMT may ultimately be more effective at preventing recurrent disease. Although many uncertainties remain, clinicians treating postoperative CD should attempt to risk-stratify all patients for postoperative recurrence before surgery and have a clear plan in place following surgery, including routine assessment for disease recurrence, regardless of the initial empiric treatment strategy.

\section{Disclosure}

BPV is supported by the National Center for Advancing Translational Sciences of the National Institutes of Health Award Number UL1TR000114. He has received research support from Genetech-Roche and speaking/consulting fees from AbbVie and Janssen. The authors report no other conflicts of interest in this work.

\section{References}

1. Peyrin-Biroulet L, Loftus EV Jr, Colombel JF, Sandborn WJ. The natural history of adult Crohn's disease in population-based cohorts. Am J Gastroenterol. 2010;105(2):289-297.

2. Bernell O, Lapidus A, Hellers G. Risk factors for surgery and postoperative recurrence in Crohn's disease. Ann Surg. 2000;231(1):38-45.

3. Olaison G, Smedh K, Sjodahl R. Natural course of Crohn's disease after ileocolic resection: endoscopically visualised ileal ulcers preceding symptoms. Gut. 1992;33(3):331-335.

4. Rutgeerts P, Geboes K, Vantrappen G, et al. Natural history of recurrent Crohn's disease at the ileocolonic anastomosis after curative surgery. Gut. 1984;25(6):665-672.

5. Rutgeerts P, Geboes K, Vantrappen G, et al. Predictability of the postoperative course of Crohn's disease. Gastroenterology. 1990;99(4):956-963.

6. Ramadas AV, Gunesh S, Thomas GA, Williams GT, Hawthorne AB. Natural history of Crohn's disease in a population-based cohort from Cardiff (1986-2003): a study of changes in medical treatment and surgical resection rates. Gut. 2010;59(9):1200-1206.

7. Peyrin-Biroulet L, Harmsen WS, Tremaine WJ, Zinsmeister AR, Sandborn WJ, Loftus EV Jr. Surgery in a population-based cohort of Crohn's disease from Olmsted County, Minnesota (1970-2004). Am J Gastroenterol. 2012;107(11):1693-1701.

8. Peng QH, Wang YF, He MQ, Zhang C, Tang Q. Clinical literature review of 1858 Crohn's disease cases requiring surgery in China. World J Gastroenterol. 2015;21(15):4735-4743.

9. Reese GE, Nanidis T, Borysiewicz C, Yamamoto T, Orchard T, Tekkis PP. The effect of smoking after surgery for Crohn's disease: a meta-analysis of observational studies. Int $J$ Colorectal Dis. 2008;23(12):1213-1221.

10. Kane SV, Flicker M, Katz-Nelson F. Tobacco use is associated with accelerated clinical recurrence of Crohn's disease after surgically induced remission. J Clin Gastroenterol. 2005;39(1):32-35.

11. McLeod RS, Wolff BG, Ross S, Parkes R, McKenzie M; Investigators of the CAST Trial. Recurrence of Crohn's disease after ileocolic resection is not affected by anastomotic type: results of a multicenter, randomized, controlled trial. Dis Colon Rectum. 2009;52(5):919-927.

12. Riss S, Schuster I, Papay P, Mittlbock M, Stift A. Repeat intestinal resections increase the risk of recurrence of Crohn's disease. Dis Colon Rectum. 2013;56(7):881-887.
13. Simillis C, Yamamoto T, Reese GE, et al. A meta-analysis comparing incidence of recurrence and indication for reoperation after surgery for perforating versus nonperforating Crohn's disease. Am J Gastroenterol. 2008;103(1):196-205.

14. Sachar DB, Lemmer E, Ibrahim C, et al. Recurrence patterns after first resection for stricturing or penetrating Crohn's disease. Inflamm Bowel Dis. 2009;15(7):1071-1075.

15. Silverberg MS, Satsangi J, Ahmad T, et al. Toward an integrated clinical, molecular and serological classification of inflammatory bowel disease: report of a Working Party of the 2005 Montreal World Congress of Gastroenterology. Can J Gastroenterol. 2005;19(Suppl A):5a-36a.

16. Ferrante M, de Hertogh G, Hlavaty T, et al. The value of myenteric plexitis to predict early postoperative Crohn's disease recurrence. Gastroenterology. 2006;130(6):1595-1606.

17. Sokol H, Polin V, Lavergne-Slove A, et al. Plexitis as a predictive factor of early postoperative clinical recurrence in Crohn's disease. Gut. 2009;58(9):1218-1225.

18. Bressenot A, Chevaux JB, Williet N, et al. Submucosal plexitis as a predictor of postoperative surgical recurrence in Crohn's disease. Inflamm Bowel Dis. 2013;19(8):1654-1661.

19. Bernell O, Lapidus A, Hellers G. Risk factors for surgery and recurrence in 907 patients with primary ileocaecal Crohn's disease. $\mathrm{Br} J$ Surg. 2000;87(12):1697-1701.

20. Welsch T, Hinz U, Loffler T, et al. Early re-laparotomy for postoperative complications is a significant risk factor for recurrence after ileocaecal resection for Crohn's disease. Int J Colorectal Dis. 2007;22(9):1043-1049.

21. De Cruz P, Kamm MA, Prideaux L, Allen PB, Desmond PV. Postoperative recurrent luminal Crohn's disease: a systematic review. Inflamm Bowel Dis. 2012;18(4):758-777.

22. Mondot S, Lepage P, Seksik P, et al. Structural robustness of the gut mucosal microbiota is associated with Crohn's disease remission after surgery. Gut. 2016;65(6):954-962.

23. De Cruz P, Kang S, Wagner J, et al. Association between specific mucosa-associated microbiota in Crohn's disease at the time of resection and subsequent disease recurrence: a pilot study. $J$ Gastroenterol Hepatol. 2015;30(2):268-278.

24. He X, Chen Z, Huang J, et al. Stapled side-to-side anastomosis might be better than handsewn end-to-end anastomosis in ileocolic resection for Crohn's disease: a meta-analysis. Dig Dis Sci. 2014;59(7):1544-1551.

25. Bressenot A, Peyrin-Biroulet L. Histologic features predicting postoperative Crohn's disease recurrence. Inflamm Bowel Dis. 2015;21(2):468-475.

26. Rahier JF, Dubuquoy L, Colombel JF, et al. Decreased lymphatic vessel density is associated with postoperative endoscopic recurrence in Crohn's disease. Inflamm Bowel Dis. 2013;19(10):2084-2090.

27. Li Y, Stocchi L, Liu X, et al. Presence of granulomas in mesenteric lymph nodes is associated with postoperative recurrence in Crohn's disease. Inflamm Bowel Dis. 2015;21(11):2613-2618.

28. Boschetti G, Nancey S, Moussata D, et al. Enrichment of circulating and mucosal cytotoxic CD8+ T cells is associated with postoperative endoscopic recurrence in patients with Crohn's disease. J Crohns Colitis. 2016;10(3):338-345.

29. Li Y, Zhu W, Gong J, et al. Visceral fat area is associated with a high risk for early postoperative recurrence in Crohn's disease. Colorectal Dis. 2015;17(3):225-234.

30. De Cruz P, Kamm MA, Hamilton AL, et al. Crohn's disease management after intestinal resection: a randomised trial. Lancet. 2015; 385(9976):1406-1417.

31. Regueiro M. Management and prevention of postoperative Crohn's disease. Inflamm Bowel Dis. 2009;15(10):1583-1590.

32. Barrie A, Regueiro M. Postoperative anti-TNF therapy in the management of Crohn's disease. In Rogler G, Herfarth H, Hibi, T, Nielsen OJ, editors. Anti-Tumor Necrosis Factor Therapy in Inflammatory Bowel Disease. Bael, Switzerland, Karger; 2015. 
33. Buisson A, Chevaux JB, Bommelaer G, Peyrin-Biroulet L. Diagnosis, prevention and treatment of postoperative Crohn's disease recurrence. Dig Liver Dis. 2012;44(6):453-460.

34. Van Assche G, Dignass A, Reinisch W, et al. The second European evidence-based Consensus on the diagnosis and management of Crohn's disease: Special situations. J Crohns Colitis. 2010;4(1):63-101.

35. Singh S, Garg SK, Pardi DS, Wang Z, Murad MH, Loftus EV Jr. Comparative efficacy of pharmacologic interventions in preventing relapse of Crohn's disease after surgery: a systematic review and network meta-analysis. Gastroenterology. 2015;148(1):64-76.e62; quiz e14.

36. Doherty G, Bennett G, Patil S, Cheifetz A, Moss AC. Interventions for prevention of post-operative recurrence of Crohn's disease. Cochrane Database Syst Rev. 2009;(4):Cd006873.

37. D'Haens GR, Vermeire S, Van Assche G, et al. Therapy of metronidazole with azathioprine to prevent postoperative recurrence of Crohn's disease: a controlled randomized trial. Gastroenterology. 2008;135(4):1123-1129.

38. Hanauer SB, Korelitz BI, Rutgeerts P, et al. Postoperative maintenance of Crohn's disease remission with 6-mercaptopurine, mesalamine, or placebo: a 2-year trial. Gastroenterology. 2004;127(3):723-729.

39. Shen FC, Zhang HJ, Zhao XD, Cao RS, Shi RH. Purine analogues compared with mesalamine or 5-ASA for the prevention of postoperative recurrence in Crohn's disease: a meta-analysis. Int J Clin Pract. 2012;66(8):758-766.

40. Reinisch W, Angelberger S, Petritsch W, et al. Azathioprine versus mesalazine for prevention of postoperative clinical recurrence in patients with Crohn's disease with endoscopic recurrence: efficacy and safety results of a randomised, double-blind, double-dummy, multicentre trial. Gut. 2010;59(6):752-759.

41. Fedorak RN, Feagan BG, Hotte N, et al. The probiotic VSL\#3 has anti-inflammatory effects and could reduce endoscopic recurrence after surgery for Crohn's disease. Clin Gastroenterol Hepatol. 2015;13(5):928-935.e922.

42. Rutgeerts P, Hiele M, Geboes K, et al. Controlled trial of metronidazole treatment for prevention of Crohn's recurrence after ileal resection. Gastroenterology. 1995;108(6):1617-1621.

43. Rutgeerts P, Van Assche G, Vermeire S, et al. Ornidazole for prophylaxis of postoperative Crohn's disease recurrence: a randomized, double-blind, placebo-controlled trial. Gastroenterology. 2005; 128(4):856-861.

44. Manosa M, Cabre E, Bernal I, et al. Addition of metronidazole to azathioprine for the prevention of postoperative recurrence of Crohn's disease: a randomized, double-blind, placebo-controlled trial. Inflamm Bowel Dis. 2013;19(9):1889-1895.

45. Ananthakrishnan AN, Hur C, Juillerat P, Korzenik JR. Strategies for the prevention of postoperative recurrence in Crohn's disease: results of a decision analysis. Am J Gastroenterol. 2011;106(11):2009-2017.

46. Zhao Y, Ma T, Chen YF, et al. Biologics for the prevention of postoperative Crohn's disease recurrence: a systematic review and meta-analysis. Clin Res Hepatol Gastroenterol. 2015;39(5):637-649.

47. Qiu Y, Mao R, Chen BL, HeY, Zeng ZR, Chen MH. Systematic review with meta-analysis of prospective studies: anti-tumour necrosis factor for prevention of postoperative Crohn's disease recurrence. J Crohns Colitis. 2015;9(10):918-927.

48. Yang Z, Ye X, Wu Q, Wu K, Fan D. A network meta-analysis on the efficacy of 5-aminosalicylates, immunomodulators and biologics for the prevention of postoperative recurrence in Crohn's disease. Int $J$ Surg. 2014;12(5):516-522.

49. Regueiro M, Feagan BG, Zou B, et al. 749 infliximab for prevention of recurrence of post-surgical Crohn's disease following ileocolonic resection: a randomized, placebo-controlled study. Gastroenterology. 2015;148(4):S-141.

50. Regueiro M, Schraut W, Baidoo L, et al. Infliximab prevents Crohn's disease recurrence after ileal resection. Gastroenterology. 2009;136(2):441-450.e441; quiz 716.
51. Yoshida K, Fukunaga K, Ikeuchi H, et al. Scheduled infliximab monotherapy to prevent recurrence of Crohn's disease following ileocolic or ileal resection: a 3-year prospective randomized open trial. Inflamm Bowel Dis. 2012;18(9):1617-1623.

52. Regueiro M, Kip KE, Baidoo L, Swoger JM, Schraut W. Postoperative therapy with infliximab prevents long-term Crohn's disease recurrence. Clin Gastroenterol Hepatol. 2014;12(9):1494-1502.e1491.

53. Armuzzi A, Felice C, Papa A, et al. Prevention of postoperative recurrence with azathioprine or infliximab in patients with Crohn's disease: an open-label pilot study. J Crohns Colitis. 2013;7(12):e623-e629.

54. Fernández-Blanco I, Monturiol J, Martinez B, Cara C, Taxonera C. W1291 adalimumab in the prevention of postoperative recurrence of Crohn's disease. Gastroenterology. 2010;138(5):S-S692.

55. Tursi A, Elisei W, Picchio M, et al. Comparison of the effectiveness of infliximab and adalimumab in preventing postoperative recurrence in patients with Crohn's disease: an open-label, pilot study. Tech Coloproctol. 2014;18(11):1041-1046.

56. Kotze PG, Yamamoto T, Danese S, et al. Direct retrospective comparison of adalimumab and infliximab in preventing early postoperative endoscopic recurrence after ileocaecal resection for crohn's disease: results from the MULTIPER database. J Crohns Colitis. 2015;9(7):541-547.

57. De Cruz P, Kamm MA, Hamilton AL, et al. Efficacy of thiopurines and adalimumab in preventing Crohn's disease recurrence in highrisk patients - a POCER study analysis. Aliment Pharmacol Ther. 2015;42(7):867-879.

58. Savarino E, Bodini G, Dulbecco P, et al. Adalimumab is more effective than azathioprine and mesalamine at preventing postoperative recurrence of Crohn's disease: a randomized controlled trial. Am J Gastroenterol. 2013;108(11):1731-1742.

59. Doherty GA, Miksad RA, Cheifetz AS, Moss AC. Comparative costeffectiveness of strategies to prevent postoperative clinical recurrence of Crohn's disease. Inflamm Bowel Dis. 2012;18(9):1608-1616.

60. Collins M, Sarter H, Gower-Rousseau C, et al. Tu1337 Anti-TNF $\alpha$ treatment efficacy in prevention of postoperative recurrence in Crohn's disease depends on previous exposure to anti-TNF $\alpha$ agents. Gastroenterology. 2015;148(4):Suppl 1:S-S863.

61. Gagniere C, Beaugerie L, Pariente B, et al. Benefit of infliximab reintroduction after successive failure of infliximab and adalimumab in Crohn's disease. J Crohns Colitis. 2015;9(4):349-355.

62. Colombel JF, Sandborn WJ, Reinisch W, et al. Infliximab, azathioprine, or combination therapy for Crohn's disease. $N$ Engl J Med. 2010; 362(15):1383-1395.

63. Sakuraba A, Okamoto S, Matsuoka K, et al. Combination therapy with infliximab and thiopurine compared to infliximab monotherapy in maintaining remission of postoperative Crohn's disease. Digestion. 2015;91(3):233-238.

64. Suzuki Y, Kotze PG, Spinelli A, et al. Su1376 retrospective comparison of the efficacy of Anti-TNF agents in isolation or combined with azathioprine in prevention of early postoperative endoscopic recurrence in Crohn's disease from the multiper database. Gastroenterology. 146(5):S-S451.

65. Zaghiyan KN, Melmed GY, Dubinsky M, et al. Su1384 medical prophylaxis after ileocolic resection for Crohn's disease \&\#x2013; no need to rush. Gastroenterology.146(5):S-S454.

66. Bordeianou L, Stein SL, Ho VP, et al. Immediate versus tailored prophylaxis to prevent symptomatic recurrences after surgery for ileocecal Crohn's disease? Surgery. 2011;149(1):72-78.

67. Angelberger S, Schaeffeler E, Teml A, et al. Mucosal improvement in patients with moderate to severe postoperative endoscopic recurrence of Crohn's disease and azathioprine metabolite levels. Inflamm Bowel Dis. 2013;19(3):590-598.

68. Bodini G, Savarino V, Peyrin-Biroulet L, et al. Low serum trough levels are associated with post-surgical recurrence in Crohn's disease patients undergoing prophylaxis with adalimumab. Dig Liver Dis. 2014;46(11): 1043-1046. 
69. Vande Casteele N, Ferrante M, Van Assche G, et al. Trough concentrations of infliximab guide dosing for patients with inflammatory bowel disease. Gastroenterology. 2015;148(7):1320-1329.e1323.

70. Vaughn BP, Martinez-Vazquez M, Patwardhan VR, et al. Proactive therapeutic concentration monitoring of infliximab may improve outcomes for patients with inflammatory bowel disease: results from a pilot observational study. Inflamm Bowel Dis. 2014;20(11):1996-2003.

71. Brandse JF, van den Brink GR, Wildenberg ME, et al. Loss of infliximab into feces is associated with lack of response to therapy in patients with severe ulcerative colitis. Gastroenterology. 2015;149(2):350-355.e352.

72. Sorrentino D, Paviotti A, Terrosu G, et al. Low-dose maintenance therapy with infliximab prevents postsurgical recurrence of Crohn's disease. Clin Gastroenterol Hepatol. 2010;8(7):591-599.e591; quiz e578-e599

73. Sorrentino D, Marino M, Dassopoulos T, Zarifi D, Del Bianco T. Low dose infliximab for prevention of postoperative recurrence of crohn's disease: long term follow-up and impact of infliximab trough levels and antibodies to infliximab. PLoS One. 2015;10(12):e0144900.

74. Regueiro M, Kip KE, Schraut W, et al. Crohn's disease activity index does not correlate with endoscopic recurrence one year after ileocolonic resection. Inflamm Bowel Dis. 2011;17(1):118-126.

75. Yamamoto T, Bamba T, Umegae S, Matsumoto K. The impact of early endoscopic lesions on the clinical course of patients following ileocolonic resection for Crohn's disease: a 5-year prospective cohort study. United European Gastroenterol J. 2013;1(4):294-298.

76. Mitselos IV, Christodoulou DK, Katsanos KH, Tsianos EV. Role of wireless capsule endoscopy in the follow-up of inflammatory bowel disease. World J Gastrointest Endosc. 2015;7(6):643-651.

77. Mao R, Gao X, Zhu ZH, et al. CT enterography in evaluating postoperative recurrence of Crohn's disease after ileocolic resection: complementary role to endoscopy. Inflamm Bowel Dis. 2013;19(5):977-982.

78. Minordi LM, Vecchioli A, Poloni G, Guidi L, De Vitis I, Bonomo L. Enteroclysis CT and PEG-CT in patients with previous small-bowel surgical resection for Crohn's disease: CT findings and correlation with endoscopy. Eur Radiol. 2009;19(10):2432-2440.

79. Gallego Ojea JC, Echarri Piudo AI, Porta Vila A. Crohn's disease: the usefulness of MR enterography in the detection of recurrence after surgery. Radiologia. 2011;53(6):552-559.

80. Soyer P, Boudiaf M, Sirol M, et al. Suspected anastomotic recurrence of Crohn disease after ileocolic resection: evaluation with CT enteroclysis. Radiology. 2010;254(3):755-764.

81. Sailer J, Peloschek P, Reinisch W, Vogelsang H, Turetschek K, Schima W. Anastomotic recurrence of Crohn's disease after ileocolic resection: comparison of MR enteroclysis with endoscopy. Eur Radiol. 2008;18(11):2512-2521.

82. Koilakou S, Sailer J, Peloschek P, et al. Endoscopy and MR enteroclysis: equivalent tools in predicting clinical recurrence in patients with Crohn's disease after ileocolic resection. Inflamm Bowel Dis. 2010;16(2):198-203.

83. Ercole E, Rigazio C. Role of bowel ultrasound in the management of postoperative Crohn's disease. World J Gastrointest Pathophysiol. 2014;5(4):457-461.

84. Lasson A, Strid H, Ohman L, et al. Fecal calprotectin one year after ileocaecal resection for Crohn's disease - a comparison with findings at ileocolonoscopy. J Crohns Colitis. 2014;8(8):789-795.

85. Yamamoto T, Shiraki M, Bamba T, Umegae S, Matsumoto K. Faecal calprotectin and lactoferrin as markers for monitoring disease activity and predicting clinical recurrence in patients with Crohn's disease after ileocolonic resection: a prospective pilot study. United European Gastroenterol J. 2013;1(5):368-374.
86. Lobaton T, Lopez-Garcia A, Rodriguez-Moranta F, Ruiz A, Rodríguez L, Guardiola J. A new rapid test for fecal calprotectin predicts endoscopic remission and postoperative recurrence in Crohn's disease. $J$ Crohns Colitis. 2013;7(12):e641-e651.

87. Qiu Y, Mao R, Chen BL, et al. Fecal calprotectin for evaluating postoperative recurrence of Crohn's disease: a meta-analysis of prospective studies. Inflamm Bowel Dis. 2015;21(2):315-322.

88. Boschetti G, Laidet M, Moussata D, et al. Levels of fecal calprotectin are associated with the severity of postoperative endoscopic recurrence in asymptomatic patients with Crohn's disease. Am J Gastroenterol. 2015;110(6):865-872.

89. Wright EK, Kamm MA, De Cruz P, et al. Measurement of fecal calprotectin improves monitoring and detection of recurrence of Crohn's disease after surgery. Gastroenterology. 2015;148(5):938-947.e931.

90. Schoepfer AM, Lewis JD. Serial fecal calprotectin measurements to detect endoscopic recurrence in postoperative Crohn's disease: is colonoscopic surveillance no longer needed? Gastroenterology. 2015;148(5):889-892.

91. Sorrentino D, Terrosu G, Paviotti A, et al. Early diagnosis and treatment of postoperative endoscopic recurrence of Crohn's disease: partial benefit by infliximab - a pilot study. Dig Dis Sci. 2012;57(5): 1341-1348.

92. Yamamoto T, Umegae S, Matsumoto K. Impact of infliximab therapy after early endoscopic recurrence following ileocolonic resection of Crohn's disease: a prospective pilot study. Inflamm Bowel Dis. 2009;15(10):1460-1466.

93. Bouguen G, Levesque BG, Feagan BG, et al. Treat to target: a proposed new paradigm for the management of Crohn's disease. Clin Gastroenterol Hepatol. 2015;13(6):1042-1050.e1042.

94. Peyrin-Biroulet L, Sandborn W, Sands BE, et al. Selecting therapeutic targets in inflammatory bowel disease (STRIDE): determining therapeutic goals for treat-to-target. Am J Gastroenterol. 2015;110(9):1324-1338.

95. Baudry C, Pariente B, Lourenco N, et al. Tailored treatment according to early post-surgery colonoscopy reduces clinical recurrence in Crohn's disease: a retrospective study. Dig Liver Dis. 2014;46(10): 887-892.

96. Gonzalez-Lama Y, Blazquez I, Suarez CJ, et al. Impact of endoscopic monitoring in postoperative Crohn's disease patients already receiving pharmacological prevention of recurrence. Rev Esp Enferm Dig. 2015;107(10):586-590.

97. Ferrante M, Papamichael K, Duricova D, et al. Systematic versus endoscopy-driven treatment with azathioprine to prevent postoperative ileal Crohn's disease recurrence. J Crohns Colitis. 2015;9(8):617-624.

98. Bryant RV, Sandborn WJ, Travis SP. Introducing vedolizumab to clinical practice: who, when, and how? J Crohns Colitis. 2015;9(4):356-366

99. Poulakos M, Machin JD, Pauly J, Grace Y. Vedolizumab: a new opponent in the battle against Crohn's disease and ulcerative colitis. J Pharm Pract. Epub May 6, 2015.

100. Targownik LE, Coneys JG, Dhillon AS. Emerging issues in the medical management of Crohn's disease. Curr Opin Gastroenterol. 2016;32(2):103-109.

101. Rutgeerts P, Goboes K, Peeters M, et al. Effect of faecal stream diversion on recurrence of Crohn's disease in the neoterminal ileum. Lancet. 1991;338(8770):771-774.

102. Vaughn BP, Moss AC. Prevention of post-operative recurrence of Crohn's disease. World J Gastroenterol. 2014;20(5):1147-1154.

103. O'connor A, Taylor J, Roberts L, et al. PTU-067 How are postoperative crohn's patients followed up? a retrospective analysis from a tertiary referral centre. Gut. 2015;64(Suppl 1):A89. 


\section{Publish your work in this journal}

Clinical and Experimental Gastroenterology is an international, peerreviewed, open access, online journal publishing original research, reports, editorials, reviews and commentaries on all aspects of gastroenterology in the clinic and laboratory. This journal is included on PubMed. The manuscript management system is completely online and includes a very quick and fair peer-review system, which is all easy to use. Visit http://www.dovepress.com/testimonials.php to read real quotes from published authors.

Submit your manuscript here: https://www.dovepress.com/clinical-and-experimental-gastroenterology-journal 